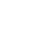
3

\title{
Protein interaction studies point to new functions for Escherichia coli glyceraldehyde-3-phosphate dehydrogenase
}

9

(1)

(1)

3

(1)

\author{
Elaine Ferreira ${ }^{\mathrm{a}}$, Rosa Giménez ${ }^{\mathrm{a}}$, Laura Aguilera ${ }^{\mathrm{a}}$, Karla Guzmán, Juan
} Aguilar ${ }^{\mathrm{a}}$, Josefa Badia ${ }^{\mathrm{a}}$, Laura Baldomà ${ }^{\mathrm{a}^{*}}$

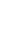

åDepartament de Bioquímica i Biología Molecular, Facultat de Farmàcia, Institut de Biomedicina de la Universitat de Barcelona (IBUB), Universitat de Barcelona, Av. Diagonal, 643. E-08028 Barcelona, Spain,

Elaine Ferreira: elainefmelo@gmail.com

Rosa Giménez: rgimenez@ub.edu

Laura Aguilera: laguilera@ub.edu

Karla Guzmán: guz_krl@hotmail.com

Juan Aguilar: juanaguilar@ub.edu

Josefa Badia: josefabadia@ub.edu

Laura Baldomà: lbaldoma@ub.edu “Correspondence and reprints” 


\section{Abstract}

Glyceraldehyde-3-phosphate dehydrogenase (GAPDH) is considered a multifunctional protein with defined functions in numerous mammalian cellular processes. GAPDH functional diversity depends on various factors such as covalent modifications, subcellular localization, oligomeric state and intracellular concentration of substrates or ligands, as well as protein-protein interactions. In bacteria, alternative GAPDH functions have been associated with its extracellular location in pathogens or probiotics. In this study, new intracellular functions of E. coli GAPDH were investigated following a proteomic approach aimed at identifying interacting partners using in vivo formaldehyde cross-linking followed by mass spectrometry. The identified proteins were involved in metabolic processes, protein synthesis and folding or DNA repair. Some interacting proteins were also identified in immunopurification experiments in the absence of cross-linking. Pull-down experiments and overlay immunoblotting were performed to further characterize the interaction with phosphoglycolate phosphatase (Gph). This enzyme is involved in the metabolism of 2-phosphoglycolate formed in the DNA repair of 3'-phosphoglycolate ends generated by bleomycin damage. We show that interaction between Gph and GAPDH increases in cells challenged with bleomycin, suggesting involvement of GAPDH in cellular processes linked to DNA repair mechanisms.

Keywords: moonlighting proteins; glyceraldehyde-3-phosphate dehydrogenase; proteinprotein interactions; phosphoglycolate phosphatase; DNA repair; Escherichia coli 
58 Abbreviations: CAA, casein acid hydrolysate CAA; CFUs, colony forming units; FA,

59 formaldehyde; GAPDH, glyceraldehyde-3-phosphate dehydrogenase; Gph,

60 phosphoglycolate phosphatase; GST, glutathione-S-transferase; MS, mass spectrometry;

61 NBRP, National BioResource Project; PVDF, polyvinylidene difluoride. 


\section{Introduction}

(2)

Glyceraldehyde-3-phosphate dehydrogenase (GAPDH, EC 1.2.1.12), a key enzyme in the glycolytic pathway, is not merely a classical metabolic protein, but is also considered a multifunctional protein with defined functions in numerous mammalian cellular processes (reviewed in Sirover, 1999, 2005, 2011). This protein is involved in transcriptional and post-transcriptional gene regulation, chromatin structure, intracellular membrane trafficking, DNA replication and DNA repair processes (Sirover, 2011). Each new function requires GAPDH association into specific protein complexes. The functional diversity of GAPDH depends on various factors, such as covalent modifications, subcellular localization, oligomeric state, or intracellular concentration of substrates (Jeffery, 2004; Sirover, 2005, 2011). In this context, it is important to consider the great diversity of posttranslational modifications described for GAPDH, which may underpin its multifunctional activity (Sirover, 2011). Many of these modifications are associated with various types of stress (Cabiscol and Ros, 2006; Colussi et al., 2000; Eaton et al., 2002).

In bacteria, alternative GAPDH functions have been associated with its extracellular location in pathogens. GAPDH is one of the housekeeping proteins that are secreted and exposed on the bacterial surface, enabling the pathogens to colonize and persist in the host (Pancholi and Chhatwal, 2003). Several reports on different gram-positive pathogens have demonstrated the ability of extracellular GAPDH to interact with various host components such as transferrin, plasminogen, extracellular matrix components or the urokinase plasminogen activator receptor (Jin et al., 2005; Schaumburg et al., 2004; Seifert et al., 2003). In the case of the gram-negative pathogens enteropathogenic E. coli (EPEC) and enterohaemorrhagic E. coli (EHEC), our group showed that secreted GAPDH is able to 
interact with human plasminogen and fibrinogen, and remains associated with Caco-2 cells upon infection (Egea et al., 2007). Extracellular GAPDH can also act as a target for oxidation and therefore may protect bacteria against host oxidative response (Aguilera et al., 2009). In recent years, GAPDH has been identified in the secretome of probiotic strains (Aguilera et al., 2012; Sanchez et al., 2009a, 2009b). Extracellular GAPDH may confer these strains an advantage in gut colonization with respect to other components of the intestinal microbiota. Besides the role of secreted GAPDH in host colonization processes, no studies of new GAPDH intracellular functions have been described so far in bacteria. In the area of systems biology, the identification of protein-protein interactions within cells is of prime importance to understand biological processes at the molecular level. Several groups have performed large-scale analyses using E. coli to identify interactions between proteins (Arifuzzaman et al., 2006; Butland et al., 2005). These studies, based on tagged protein baits that allow affinity purification of the interacting proteins, have shown that GAPDH interacts with a range of proteins, including metabolic enzymes and proteins involved in transcription or protein synthesis, suggesting the potential role of $E$. coli GAPDH in some cellular processes similar to those described in humans.

When using these affinity-based methods, transient interactions or weakly binding proteins can be lost during washing procedures. As an alternative, in vivo chemical crosslinking experiments can be performed to covalently fix interaction partners in living cells, thus preventing the loss of specific components of a protein complex prior to purification (Sinz, 2010). Although formaldehyde is widely used as a cross-linker in chromatin immunoprecipitation experiments, the number of studies which have used this reagent in combination with mass spectrometry (MS) to investigate protein-protein interaction is 
110 limited (Sinz, 2010). Examples of this approach are the identification of new proteins

111 interacting with a constitutive active form of M-Ras (Vasilescu et al., 2004) or the

112 identification of glutamate dehydrogenase as a protein that interacts with the regulator GltC

113 in Bacillus subtilis (Herberg et al., 2007).

114 To explore new GAPDH functions in E. coli, in this study we conducted in vivo

115 formaldehyde cross-linking experiments followed by MS to identify proteins interacting

116 with V5-tagged GAPDH in E. coli. We also characterized interaction with

117 phosphoglycolate phosphatase (Gph), an enzyme involved in the metabolism of 2-

118 phosphoglycolate, a compound formed in the DNA repair of 3'-phosphoglycolate ends

119 generated by bleomycin damage (Pellicer et al., 2003).

120

121

122

\section{Materials and methods}

123

2.1. Bacterial strains and growth conditions

125

The genotypes and sources of the E. coli strains used in this study are as follows. Strain MC4100 (F- araD $\Delta\left(\operatorname{argF-lac)} \operatorname{rpsL}\left(\mathrm{Str}^{\mathrm{r}}\right)\right.$ relA fihD deoC ptsF rbs) (Casadaban, 1976) was the source of chromosomal DNA used as a template for PCR amplification of gene gapA.

128 The mutant strain JA210 (gph::Kan) was derived from MC4100 (Pellicer et al., 2003).

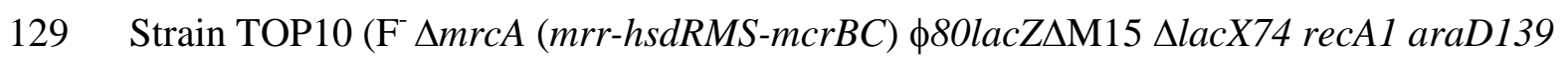

$130 \Delta$ (ara-leu)7697 galU galK $\operatorname{rpsL}\left(\mathrm{Str}^{\mathrm{r}}\right)$ endA1 nupG) was used to express recombinant

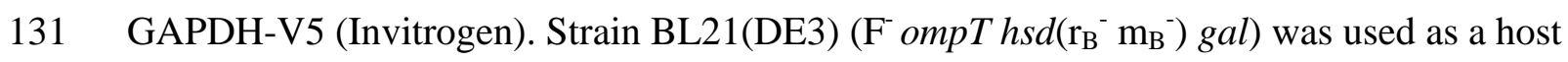

132 to express GST-tagged proteins (Amershan Pharmacia). Strain AG1 (ME5053) (recA1 
133 endA1 gyrA96 thi-1 hsdR17( $\left.\mathrm{r}_{\mathrm{K}}{ }^{-} \mathrm{m}_{\mathrm{K}}{ }^{-}\right)$supE44 realA1) was the host for recombinant

134 pCA24N plasmids expressing His-tagged proteins (NBRP, National BioResource Project).

135 Bacterial cells were routinely grown at $37^{\circ} \mathrm{C}$ in Luria-Bertani broth (LB). In bleomycin

136 challenge experiments, cells were grown in minimal medium with $0.5 \%$ casein acid

137 hydrolysate (CAA) as carbon source (Pellicer et al., 2003). Growth was monitored by

138 measuring the optical density at $600 \mathrm{~nm}\left(\mathrm{OD}_{600}\right)$. When required, tetracycline $(12.5 \mu \mathrm{g} / \mathrm{ml})$,

139 chloramphenicol (30 $\mu \mathrm{g} / \mathrm{ml})$ or ampicillin $(100 \mu \mathrm{g} / \mathrm{ml})$ was added to the medium.

140

141 2.2. Recombinant DNA techniques and site-directed mutagenesis

142 Bacterial genomic DNA was obtained using the Wizard Genomic DNA purification kit

143 (Promega), and plasmid DNA was prepared using the Wizard Plus SV Midipreps DNA

144 purification system (Promega). DNA manipulations were performed essentially as

145 described elsewhere (Sambrook and Rusell, 2001). DNA fragments were amplified by PCR

146 using E. coli chromosomal DNA as a template. PCR reactions were performed with Taq

147 DNA polymerase or $p f u$ DNA polymerase under standard conditions. DNA sequencing was

148 carried out with an automated ABI 377 DNA sequencer and fluorescent dye termination

149 methods.

150

151 2.3. Cloning of recombinant V5-tagged GAPDH

152 To construct recombinant GAPDH-V5, the pBAD TOPO® TA Expression Kit

153 (Invitrogene) was used. This system provides a highly efficient, one-step cloning strategy

154 for the direct insertion of Taq polymerase-amplified PCR products into a plasmid vector for

155 regulated expression in E. coli. Expression is driven by the araBAD promoter, thus 
156 induction of the cloned gene is achieved by the addition of L-arabinose to the culture

157 medium. The gapA gene from strain MC4100 was amplified by PCR with primers gapA-

158 pBAD-fw (TAGGGTGGAATATATGACTATCAAAGTAGG) and gapA-pBAD-rv

159 (TTTGGA GATGTGAGCGATCAGG). To express GAPDH with its native N-terminal

160 end (without the N-terminal leader), the forward primer was designed with an in-frame stop

161 codon (TAG, underlined) at the 5'-end followed by the native gapA sequence including the

162 ribosome binding site and the start codon (in bold). The gapA stop codon was removed

163 from the reverse primer to allow expression of GAPDH fused at its C-terminal end to the

164 V5 epitope. The PCR fragment was cloned into plasmid pBAD-TOPO (Invitrogen) and

165 after transformation of strain TOP10, recombinant colonies were selected on LB- ampicillin

166 plates. Plasmid DNA was sequenced to ensure that the fragment was inserted in the correct

167 orientation and that no mutations were introduced. The recombinant plasmid was named

168 pBAD-GapA.

169

170

2.4. In vivo cross-linking with formaldehyde

171

Cells of strain TOP10 bearing the recombinant plasmid pBAD-GapA were grown at

172

$37^{\circ} \mathrm{C}$ in $10 \mathrm{ml}$ of $\mathrm{LB}$-ampicillin until the culture reached an $\mathrm{OD}_{600}$ of 0.5 . At this point $\mathrm{L}-$

173

arabinose was added at a final concentration of $0.02 \%$ to induce the expression of GAPDH-

174

V5. After a $3 \mathrm{~h}$ induction, formaldehyde was added at a final concentration of $1 \%$.

175

Formaldehyde cross-linking was allowed to proceed for $20 \mathrm{~min}$ at $37^{\circ} \mathrm{C}$. To stop the cross-

176 linking reaction, glycine was added at a final concentration of $0.5 \mathrm{M}$ and after $5 \mathrm{~min}$ at room temperature, bacterial cells were harvested by centrifugation, washed twice with PBS

$178\left(140 \mathrm{mM} \mathrm{NaCl}, 2.7 \mathrm{mM} \mathrm{KCl}, 10 \mathrm{mM} \mathrm{Na}_{2} \mathrm{HPO}_{4}, 1.8 \mathrm{mM} \mathrm{KH}_{2} \mathrm{PO}_{4}, \mathrm{pH}\right.$ 7.3), and 
180 Diagnostic). Cell extracts were obtained by sonic disruption of bacterial cells followed by

181 centrifugation to remove cell debris. Protein concentration was determined using the

182 method described by Lowry et al. (1951) with bovine serum as a standard.

183

184

185

186

187

188

189

190

191

192

193

194

195

196

197

198

199

\subsection{Immunoprecipitation of recombinant GAPDH-V5}

Purification of GAPDH-V5 cross-linked complexes was performed by immunoprecipitation using anti-V5 agarose beads (Sigma-Aldrich, Germany). To reduce unspecific interactions, the binding and washing steps were carried out under astringent conditions with RIPA buffer (1\% sodium deoxycholate, 0.1\% SDS, 1\% Triton X-100, 0.14 M NaCl, 0.01 M Tris-HCl, pH 8.0). Cell extracts were precleared with protein G-agarose beads for $10 \mathrm{~min}$ at $4^{\circ} \mathrm{C}$. Precleared cell extracts containing $1.4 \mathrm{mg}$ of protein were adjusted to a final volume of $0.8 \mathrm{ml}$ with RIPA buffer and incubated with $250 \mu \mathrm{l}$ of anti-V5 beads for 90 min at $4^{\circ} \mathrm{C}$ in an orbital shaker. After five washes with RIPA buffer and two additional washes with TBS buffer (20 mM Tris-HCl, $150 \mathrm{mM} \mathrm{NaCl}, \mathrm{pH}$ 7.6), the bound complexes were eluted with $0.1 \mathrm{M}$ glycine (pH 2.5) for $15 \mathrm{~min}$ at $37^{\circ} \mathrm{C}$ and neutralized with a saturated Tris base solution. Protein from the eluted fractions was precipitated with acetone (at room temperature to minimize SDS precipitation), rinsed three times in acetone, and resuspended in loading buffer. Samples were then heated for $30 \mathrm{~min}$ at $95^{\circ} \mathrm{C}$ to reverse the formaldehyde cross-links. Proteins were separated on a 10\% SDS-polyacrylamide gel electrophoresis (SDS-PAGE) (Laemmli, 1970) and visualized by Sypro® Ruby staining. Immunoprecipitation experiments with anti-V5 agarose beads were also carried out to identify proteins that interact with GAPDH without previous cross-linking. In this case, precleared cell extracts containing $1.4 \mathrm{mg}$ of protein were adjusted to a final volume of 0.8 
203 ml with PBS buffer and incubated with anti-V5 beads as described above. After five

204 washes with PBS buffer, proteins were eluted with $0.1 \mathrm{M}$ glycine ( $\mathrm{pH}$ 2.5) for $15 \mathrm{~min}$ at

$20537^{\circ} \mathrm{C}$, neutralized and precipitated by incubation on ice with $10 \%$ trichloroacetic acid

206 (TCA). The protein pellet was washed in 90\% (v/v) ice-cold acetone, air-dried and

207 suspended in rehydration buffer (9M urea, 4\% CHAPS, $50 \mathrm{mM}$ dithiothreitol, 0.5\%

208 immobilized pH gradient buffer and traces of bromophenol blue) and processed for two-

209 dimensional gel electrophoresis.

\subsection{Two-dimensional gel electrophoresis}

2D gel electrophoresis was performed using the Protean IEF-Cell (Bio-Rad). Appropriate volumes of protein samples ( $5 \mu \mathrm{g}$ of purified recombinant GAPDH) were diluted in $125 \mu \mathrm{l}$ of rehydration buffer (9 M urea, 4\% CHAPS, $50 \mathrm{mM}$ dithiothreitol, 0.5\% immobilized $\mathrm{pH}$ gradient buffer and traces of bromophenol blue). Isoelectric focusing was performed in immobilized pH 3-10 or pH 5-8 gradient strips (BioRad). Second dimension SDS-PAGE was performed on $12.5 \%$ acrylamide gels that were processed for immunoblotting analysis. Parallel gels were silver-stained to visualize total protein.

\subsection{Mass spectrometry of proteins}

221 Following SDS-PAGE, protein bands of interest were excised from the gel and 222 sequentially washed with $25 \mathrm{mM}$ ammonium bicarbonate and acetonitrile. These samples 223 were then reduced ( $10 \mathrm{mM}$ DTT, $30 \mathrm{~min}$ at $56^{\circ} \mathrm{C}$ ), alkylated ( $55 \mathrm{mM}$ iodoacetamide, 15 224 min at $21^{\circ} \mathrm{C}$ ) and digested overnight with tripsin (Tripsin Gold, Promega) at $37^{\circ} \mathrm{C}$ in an automatic Investigator ProGest robot (Genomic Solutions). Peptides were extracted with 
$22610 \%$ formic acid and analysed by combined liquid chromatography / tandem mass

227 spectrometry (Cap-LC-nano-ESI-Q-TOF) (CapLC, Micromass-Waters) at the Barcelona

228 Science Park (PCB) Proteomics Platform. Data were generated in PKL file format and

229 submitted to a MASCOT server for database searching.

230 Protein spots from 2D gels were analysed using the liquid chromatograph nanoAcquity

231 (Waters) coupled to a mass spectrometer (OrbitrapVelos, Thermo Scientific). Raw data

232 were obtained with Thermo Xcalibur (v.2.1.0.1140) and used for database searching with

233 the Proteome Discoverer software package (v.1.2.0.124).

\subsection{Expression and purification of recombinant proteins}

Recombinant GAPDH and Gph were expressed and purified using the Glutathione-Stransferase (GST) gene fusion system with recognition sites for factor Xa cleavage as

238 described in detail previously (Egea et al., 2007; Pellicer et al., 2003).

239 When indicated, Gph was expressed as His 6 -Gph from the ASKA clone JW3348

240 (NBRP, E. coli Strain National BioResource Project) and purified under native conditions

241 with $\mathrm{Ni}^{2+}$-nitrilotriacetic acid (NTA) resin (Qiagen), as described elsewhere (Kitagawa et

242 al., 2005). The same procedure was applied for expression and purification of the ATP

243 synthase $\beta$ subunit ( His $_{6}$-AtpD) and the ATP synthase $\alpha$ subunit (His ${ }_{6}$-AtpA) from the

244 ASKA clones JW3710 and JW3712, respectively. Due to the low level expression of

245 soluble $\mathrm{His}_{6}$-AtpD, purification of this protein was achieved from a $100 \mathrm{ml}$ culture of strain

246 AG1 after overnight induction with $0.1 \mathrm{mM}$ IPTG at $20^{\circ} \mathrm{C}$. Cell pellets were suspended in 1

$247 \mathrm{ml}$ of $50 \mathrm{mM}$ sodium phosphate buffer (pH 8.0) containing $300 \mathrm{mM} \mathrm{NaCl}$ and $10 \mathrm{mM}$

248 imidazole, and sonicated on ice. The cell lysate was centrifuged at $15000 \mathrm{~g}$ and the 
249 supernatant incubated with $0.1 \mathrm{ml}$ of $\mathrm{Ni}^{2+}$-NTA resin for $1 \mathrm{~h}$ at $4^{\circ} \mathrm{C}$ with gentle shaking.

250 After loading the mixture into a column, the resin was washed with the same buffer but 251 containing $20 \mathrm{mM}$ imidazole. Recombinant proteins were eluted with $0.1 \mathrm{ml}$ of elution 252 buffer containing $300 \mathrm{mM}$ imidazole, followed by SDS-PAGE and immunoblotting 253 analysis.

\subsection{Immunoblotting analysis}

For Western blot analysis, protein samples were separated on 10\% SDS-PAGE and transferred to a HyBond-P polyvinylidene difluoride (PVDF) membrane by using a BioRad MiniTransblot apparatus. The membrane was blocked in PBS-0.05\% Tween-20 and 5\% skimmed milk (blocking solution) for $1 \mathrm{~h}$ at room temperature, incubated with primary specific antibodies against GAPDH (Egea et al., 2007) (1:5,000 dilution in blocking solution) for $16 \mathrm{~h}$ at $4^{\circ} \mathrm{C}$, washed four times with PBS-0.05\% Tween-20 and incubated with the secondary antibody (donkey anti-rabbit immunoglobulin horseradish peroxidase-linked, diluted 1:15,000 in blocking solution). Incubation with the secondary antibody was omitted when anti-V5 (Invitrogen; 1:15,000 dilution) or anti-GST (GenScript; 1:5,000 dilution) horseradish peroxidase-linked antibodies were used as primary antibody. The proteinantibody complex was visualized using the ECL Plus Western blotting detection system (Amersham Pharmacia Biotech). described above. After being blocked overnight with 1\% gelatin in TBS buffer, the membrane was incubated with purified $\mathrm{Gph}(5 \mu \mathrm{g} / \mathrm{ml})$ diluted in the same blocking buffer 
272 for 3 hours and then washed four times in TBS-0.05\% Tween-20. To visualize interaction

273 of GAPDH with Gph, the membrane was incubated with anti-Gph specific antibodies

274 (1:5,000 dilution TBS-1\% gelatin-0.05\% Tween-20) for $16 \mathrm{~h}$ at $4^{\circ} \mathrm{C}$, and processed as 275 described above using the ECL Western blotting kit to visualize the reactive spots. The 276 incubation step with purified Gph was omitted as negative control.

277 The anti-Gph antibodies used in this study were purified by affinity chromatography.

278 For this purpose, purified Gph was covalently linked to Ultralink ${ }^{\mathrm{TM}}$ Immobilized DADPA

279 on 3 M Emphase Biosupport medium AB1 gel (Pierce) using water-soluble 1-ethyl-3-(3-

280 dimethylamino-propyl)carbodiimide-HCl. To purify the anti-Gph immunoglobulin, $3 \mathrm{ml}$ of

281 the globulin fraction partially purified from the Gph-antisera (Pellicer et al., 2003) was

282 applied to the DADPA-Gph affinity column and the bound antibodies were eluted with 0.1

283 M glycine buffer ( $\mathrm{pH}$ 2.5). Collected fractions were neutralized with Tris solution ( $\mathrm{pH} 10$ )

284 and dialysed overnight against PBS.

285

286 2.10. Bleomycin cell treatment

287 Cells of strain JA210 bearing plasmid pGEX-Gph (Pellicer et al., 2003) were grown 288 aerobically in minimal medium with $0.5 \%$ CAA to mid-exponential phase $\left(\mathrm{OD}_{600}\right.$ of 0.5$)$.

289 After a 2 hour induction of GST-Gph expression with 0.5 mM IPTG, the culture was

290 treated with $8 \mu \mathrm{g} / \mathrm{ml}$ bleomycin in the presence of $50 \mu \mathrm{M} \mathrm{FeSO}_{4}$. At different times,

291 aliquots of the culture were collected, washed twice with minimal medium and processed to

292 obtain the cell extracts. Interaction between Gph and GAPDH was evaluated by pull-down

293 experiments followed by Western blot analysis with antibodies against GAPDH. Survival

294 was estimated by diluting cells in the same medium, followed by plating on LB-ampicillin 
295 plates to determine the number of colony forming units (CFUs). Bleomycin sulphate

296 (Almirall Prodesfarma, Barcelona, Spain) was freshly prepared with $50 \mathrm{mM}$ phosphate buffer (pH 7.5). $\mathrm{FeSO}_{4}$ solutions were prepared immediately before use.

\section{Results and discussion}

\subsection{Immunoaffinity purification of proteins cross-linked to GAPDH-V5 and their} formaldehyde and the GAPDH-V5 containing complexes were analysed in the cell extracts by Western blotting with either anti-V5 or anti-GAPDH antibodies. As control, TOP10

307 cells without the recombinant pBAD-GapA plasmid were processed in parallel. As shown in Fig. 1A, protein bands ranging in size from $70 \mathrm{kDa}$ to $160 \mathrm{kDa}$ were only detected above recombinant GAPDH-V5 (37 kDa) in cells treated with the cross-linker. The most apparent bands corresponded to high molecular protein complexes in accordance with the native

311 GAPDH tetramer structure. The presence of protein complexes with a molecular mass lower than $160 \mathrm{kDa}$ indicates that other oligomeric or monomeric GAPDH-V5 forms were

313 being cross-linked with $E$. coli proteins. To set up the conditions for cross-linking reversal, 314 cell extracts were incubated at $65^{\circ} \mathrm{C}$ or $95^{\circ} \mathrm{C}$ in loading buffer and at the indicated times, 315 reversion of the cross-links was analysed by Western blotting with anti-V5 antibodies (Fig. 316 1B). The high molecular mass complexes produced by formaldehyde treatment were no 317 longer detected after $30 \mathrm{~min}$ incubation at $95^{\circ} \mathrm{C}$. These conditions were selected for further 318 experiments. 
In order to identify the proteins that were cross-linked to GAPDH-V5, precleared

320 cell extracts obtained from TOP10 (pBAD-GapA) cells challenged with formaldehyde were 321 incubated with anti-V5 beads. After astringent washing steps, bound material was eluted as described in section 2.5. Cell extracts obtained from non formaldehyde-treated TOP10 (pBAD-GapA) cells were processed in parallel. Purified complexes were concentrated by acetone precipitation, resuspended in loading buffer and processed to reverse formaldehyde cross-links. Proteins were separated by SDS-PAGE. Staining with Sypro® Ruby revealed several proteins in the cross-linked sample that were not visible in the lane corresponding to non-treated cells (Fig. 2). These differential proteins were selected for further analysis. In the absence of formaldehyde treatment, co-purification of native GAPDH (band 2) with recombinant GAPDH-V5 (band 3) under astringent conditions (Fig. 2, - FA lane, Table 1) indicated a strong interaction of GAPDH monomers compatible with the stable tetrameric 331 structure reported for this protein. Twelve major bands were excised from the cross-linked sample (Fig. 2, + FA lane) and digested with trypsin. Peptides were extracted and analysed by combined liquid chromatography / tandem mass spectrometry (Cap-LC-nano-ESI-Q-TOF). Database searching in MASCOT resulted in the identification of 16 proteins, each of which matched to at least 3 unique peptide sequences (Table 1). Among the identified proteins, there were several metabolic enzymes, chaperones and factors involved in protein synthesis.

339 appeared as candidates for interaction with GAPDH in other studies of E. coli or in 340 databases such as DIP (database of interacting proteins) or IntAct. The chaperones DnaK 341 (P0A6Y8) and trigger factor (P0A850) were identified in large-scale analyses 342 (Arifuzzaman et al., 2006; Butland et al., 2005). Trigger factor interacts with the large 
343 ribosomal subunit near the peptide exit channel and binds to nascent polypeptides to assist 344 cotranslational protein folding. Therefore, interaction of GAPDH-V5 with this chaperone is 345 likely to occur during the synthesis of the recombinant polypeptide. In addition, trigger 346 factor has been shown to prevent aggregation and promote refolding of denatured GAPDH 347 in vitro (Huang et al., 2000). GAPDH has also been identified as a substrate of DnaK 348 (Deuerling et al., 2003). Both trigger factor and DnaK share a common substrate pool in 349 vivo and cooperate to ensure proper folding of the cytosolic proteins (Deuerling et al., 1999, 350 2003). Thus, interaction between the synthesized recombinant protein and these chaperones 351 is expected to occur in cells grown at $37^{\circ} \mathrm{C}$. GAPDH was identified as a putative interacting partner of Gph (P32662) in the 353 large-scale study performed by Arifuzzaman et al. (2006). In this case, the interaction was 354 observed with $\mathrm{His}_{6}$-Gph used as the bait in pull-down experiments. Regarding the 355 interaction with AldA (P25553), information available on the IntAct database 356 http://www.ebi.ac.uk/ intact/pages/interactions/ interactions) corroborates our results. Both 357 GAPDH and AldA were identified together with L-asparaginase-2 (P00805) as partners of 358 a protein complex resolved by native-PAGE. The other proteins identified in this study as putative partners of GAPDH interactions (Table 1) have not been experimentally isolated in previous studies. However, some of them are predicted to be functional partners of GAPDH in the STRING interaction network (http://string-db.org/newstring_cgi/ show_network_section.pl). Enolase and

363 transketolase belong to this group. Other proteins may establish weak or transient

364 interactions with GAPDH, being captured in our experimental conditions by formaldehyde 365 cross-linking. Alternatively, some interactions may be indirect through association of these 366 proteins as partners of multiprotein complexes. 
3.2. Identification of proteins that co-purify with GAPDH-V5 in the absence of crosslinking

Immunoprecipitation experiments with anti-V5 agarose beads were also carried out to identify proteins that interact with GAPDH without previous cross-linking. Precleared cell extracts were processed as described in section 2.5. Purified proteins were concentrated by TCA precipitation, resuspended in rehydration buffer and processed for $2 \mathrm{D}$ gel electrophoresis. In addition to the dominant spots corresponding to recombinant GAPDHV5 and native GAPDH forms, staining with Sypro ${ }^{\circledR}$ Ruby also revealed other proteins that co-purified with GAPDH-V5 under these conditions (Fig. 2B). An immunoprecipitation experiment starting with precleared cell extracts of TOP10 cells was performed as control. In the absence of a V5-tagged protein, no apparent spots were visible (not shown), thus ruling out co-purification of these proteins through interactions with the affinity matrix. Ten spots were excised from the 2D gel (Fig. 2B) and analysed by LC-MS/MS, in this case using a high sensitivity OrbitrapVelos mass spectrometer that enables identification of proteins even at very low amounts. Results are summarized in Table 2. Among these proteins, the ATP synthase $\beta$ subunit (AtpD) and the porin OmpC, both displaying high scores in this analysis, were also identified as proteins interacting with GAPDH after formaldehyde cross-linking (Table 1). Regarding the ATP synthase $\alpha$ subunit (AtpA) and LpdA (both in spot 1), although these proteins were not listed in Table 1, the MS analysis performed in the cross-linking experiment revealed in both cases two peptides matching these sequences. Another protein found here to co-purify with GAPDH was GatY (30.8 kDa), the catalytic subunit of D-tagatose-1,6-bisphosphate aldolase (Brinkkötter et al., 2002). Large-scale proteomic studies performed by Burtland et al. (2005) identified the 
regulatory subunit GatZ as an interacting partner of GAPDH. Neither GatY nor GatZ were identified as interacting with GAPDH in the cross-linking experiment. However, it should be noted that in this experiment, only proteins specifically present in the FA-treated sample were excised and analysed by MS. In this sense, GatY should correspond to one of the nonexcised protein bands with a molecular mass around $30 \mathrm{kDa}$, also visible in the control sample (Fig. 1A lane -FA versus +FA).

The protein displaying the highest score in this analysis was AtpD, with 40 peptides identified that covered $85.87 \%$ of the sequence (Table 2). This ATP synthase subunit interacts with the $\alpha$ subunit (AtpA) to form the catalytic domain for ATP synthesis. AtpA also co-purified with GAPDH-V5, although the MS analysis yielded a lower score. In this case, 29 peptides were identified that covered $61.79 \%$ of the sequence (Table 2). To validate interaction of GAPDH with both ATP synthase subunits, pull-down experiments with $\mathrm{His}_{6}$-tagged AtpD and AtpA proteins were performed. Recombinant His $_{6}{ }^{-}$ AtpD and His $_{6}$-AtpA were expressed and purified from the ASKA clones JW3710 and JW3712, respectively, as described in section 2.7. Immunoblotting of the column fractions with anti-GAPDH antibodies indicated that a fraction of GAPDH was bound and co-eluted with either $\mathrm{His}_{6}$-AtpD or $\mathrm{His}_{6}$-AtpA (Fig. 3). In a control experiment, the cell extract obtained from the host strain AG1 was directly applied to the $\mathrm{Ni}^{2+}$-NTA column and processed in parallel as a control to confirm that the $\mathrm{Ni}^{2+}$-NTA resin did not bind GAPDH to any extent (Fig. 3, right panel). It is noteworthy that even at lower bait protein concentration the GAPDH fraction recovered in these experiments was higher in the case of His $_{6}$-AtpD. This suggests that co-purification and binding of GAPDH to AtpA may be 
412 indirect through its interaction with the AtpD subunit. Alternatively, association of the

413 AtpD and AtpA may be required to provide the interaction domain with GAPDH. It should be noted that in addition to AtpA, spot 1 contained other ATP-binding proteins such as AraG and RbsA. Abundance of these proteins in the spot should be very low given the low score and number of sequenced peptides (Table 2). Their identification may be attributed to the high sensitivity achieved with the analysis through use of the OrbitrapVelos mass spectrometer. All the proteins identified in spot 1 displayed similar molecular mass and pI, which is compatible with their inclusion in a single spot (Table 2). The fact that out of these five proteins, three contained ATP-binding domains suggests that 421 GAPDH may display affinity for such domains. These kinds of interaction may point to a 422 regulatory role of GAPDH in energy-dependent processes. One such process is cell 423 motility. In this context, GAPDH has been suggested to play an important role in the 424 motility of the halotolerant alga Dunaliella salina. In this alga, GAPDH was found in the 425 flagellar proteome; in addition, silencing of GAPDH expression by RNAi led to reduced motility (Jia et al., 2009).

A relationship between GAPDH and cellular processes involved in energy production and conversion has been observed in Streptococcus pyogenes (Jin et al., 2011). Besides its cytoplasmic location, surface export of GAPDH is essential for Streptococcus virulence.

430 Mutants unable to export this protein, and thus expressing higher intracellular GAPDH 431 levels, displayed an 8-fold increase in the intracellular ATP concentration with respect to 432 the wild-type strain. Moreover, this study revealed that intracellular levels of GAPDH in 433 some way control the expression of certain genes (Jin et al., 2011). For instance, microarray 434 analysis of this mutant revealed up-regulation of genes encoding proton-translocating 435 ATPases. In this context, the interaction of GAPDH with ATP synthase subunits identified 
436 here suggests that there are additional mechanisms in the regulatory role of GAPDH in

437 ATP-dependent processes.

438

439

\subsection{Analysis of GAPDH interaction with Gph}

Among the proteins identified as potential GAPDH interacting partners, we found

441 the interaction with Gph to be of special interest due to its physiological role in processes

442 linked to DNA repair. Our group showed for the first time in bacteria that this

443 housekeeping enzyme is involved in the dissimilation of the intracellular 2-

444 phosphoglycolate formed in the DNA repair of 3'-phosphoglycolate ends (Pellicer et al., 445 2003). These kinds of DNA strand break are caused by bleomycin (Povirk, 1996). In

446 mammals, involvement of GAPDH in DNA repair processes has been well-documented

447 (Sirover, 2005; Azam et al., 2008); however no reports on a similar function were available 448 for prokaryotic GAPDH.

449 First, we aimed to determine whether GAPDH copurified with recombinant Gph. To 450 this end, cell extracts of strain BL21 bearing plasmid pGEX-Gph, and therefore expressing 451 GST-Gph (Pellicer et al., 2003), were applied to a glutathione-sepharose 4B resin. Cell 452 extracts expressing the tag protein GST were processed in parallel as control. After 453 extensive washing, elution of GST or GST-Gph was performed with glutathione elution 454 buffer. Western blot analysis of the eluted fractions indicated that GAPDH co-purified with 455 GST-Gph but not with GST (Fig. 4A). In a parallel experiment, elution of Gph from the 456 affinity resin was achieved by incubation with factor Xa. Western blot analysis confirmed 457 co-elution of GAPDH (Fig. 4B). In the eluted fractions, in addition to Gph (27 kDa), two 458 other proteins were visible. The $34 \mathrm{kDa}$ protein is one of the factor Xa subunits. The 35 459 kDa protein was excised from the gel and identified as GAPDH by MS. Moreover, pull- 
460

461

down experiments using His $_{6}$-Gph expressed in strain AG1 from the ASKA clone JW3348 as bait confirmed co-purification of GAPDH with Gph (not shown). Overall these results confirmed interaction of GAPDH with Gph. However, when purified GAPDH was applied to an immobilized GST-Gph column, GAPDH was scarcely retained (not shown). Most of the GAPDH protein appeared in the flow-through fraction, suggesting that interaction between these proteins may either depend on other cellular factors (proteins or ligands) or on post-translational modifications of these proteins.

The presence of multiple covalently modified forms of GAPDH (Aguilera et al., 2009; Egea et al., 2007) prompted us to analyse whether the Gph binding activity is specifically linked to one of these forms. This was determined by Far-Western experiments in which purified GAPDH was subjected to 2D gel electrophoresis. Once electroblotted, the membrane was reacted with purified Gph followed by incubation with specific anti-Gph antibodies. Of the multiple GAPDH spots, only one was visualized (Fig. 3C), indicating that Gph mainly interacts with a specific form of GAPDH. These results provide evidence of a direct interaction between both proteins, and indicate that it depends on a given posttranslational modification of GAPDH.

Since human GAPDH has been shown to be involved in repairing the DNA damage generated by bleomycin or alkylating agents (Azam et al., 2008), we examined whether Gph-GAPDH interacting complexes increased in cells challenged with bleomycin. To this end, induced exponential cultures of the gph mutant strain JA210 harbouring plasmid pGEX-Gph were treated with $8 \mu \mathrm{g} / \mathrm{ml}$ bleomycin in the presence of $50 \mu \mathrm{M} \mathrm{FeSO}_{4}$, conditions that increase the number of DNA strand breaks to be repaired. At different times, aliquots of the culture were collected and processed to obtain the cell extracts. After 
48330 min exposure to bleomycin, the percentage of viable cells was found to be around 30\%.

484 Cell extracts were incubated with glutathione-sepharose 4B resin in PBS buffer. After 485 extensive washing, GST-Gph was eluted with SDS-PAGE loading buffer. To evaluate 486 GAPDH association, fractions were analysed by Western blotting with antibodies against 487 GAPDH. For normalization, the same fractions were analysed in parallel with anti-GST 488 antibodies (Fig. 5). In these experiments, the total amount of protein applied to the 489 electrophoresis gel was lower than that used in the pull-down experiments presented in Fig. 4903 in order to avoid saturation of the luminescent signal in the immunoblotting analysis. As 491 shown in Fig. 5, the number of GAPDH molecules in complex with Gph increased in 492 bleomycin-treated cells in a time-dependent manner. Overall, these results suggest that 493 interaction between both GAPDH and Gph is triggered under activated DNA repair 494 conditions. These proteins are probably partners of protein complexes involved in such 495 processes.

Proteomic studies aimed at identifying protein interactions have great potential for suggesting new functions for a given protein. In fact, the presence of a protein in a multiprotein complex may be indicative that this protein has a second function (Jeffery, 2009). The study presented here may provide valuable clues to identify moonlighting functions for E.coli GAPDH. In this sense, the interaction with Gph points to a GAPDH role in cellular processes linked to DNA repair. In addition, interaction between these proteins may also suggest alternative GAPDH functions in quorum sensing signalling, 503 since in enteric bacteria 2-phosphoglycolate is also generated as a degradation product of 504 4,5-dihydroxy-2,3-pentanedione (DPD), the precursor of the autoinducer-2 (Taga, 2007; 505 Xavier et al., 2007). In conclusion, this study may contribute to the identification of further 506 moonlighting functions of E. coli GAPDH at the intracellular level. 
507 Acknowledgments

508

509 This research was supported by grant BFU 2010-22260-C02-01 from the Spanish

510 Ministry of Education and Science to L.B. We acknowledge NBRP-E.coli at the National

511 Institute of Genetics for providing strain AG1 and the ASKA clones JW3348, JW3710 and

512 JW3712. We thank E. Oliveira and M.A Odena for technical support in proteomic analysis.

513 


\section{References}

Aguilera, L., Giménez, R., Badia, J., Aguilar, J., Baldomà, L., 2009. NAD+'-dependent posttranslational modification of Escherichia coli glyceraldehyde-3-phosphate dehydrogenase. Int. Microbiol. 12, 187-192.

Aguilera, L., Ferreira, E., Giménez, R., Fernández, F.J., Taulés, M., Aguilar, J., Vega, M.C., Badia, J., Baldomà, L., 2012. Secretion of the housekeeping protein glyceraldehyde-3-phosphate dehydrogenase by the LEE-encoded type III secretion system in enteropathogenic Escherichia coli. Int. J. Biochem. Cell Biol. 44, 955962.

Arifuzzaman, M., Maeda, M., Itoh, A., Nishikata, K., Takita, C., Saito, R., Ara, T., Nakahigashi, K., Huang, H-C., Hirai, A., Tsuzuki, K., Nakamura, S., Altaf-UlAmin, M., Oshima, T., Baba, T., Yamamoto, N., Kawamura, T., Ioka-Nakamichi, T., Kitagawa, M., Tomita, M., Kanaya, S., Wada, C., Mori, H., 2006. Large-scale identification of protein-protein interaction of Escherichia coli K-12. Genome Res. $16,686-91$.

Azam, S., Jouvert, N., Jilani, A., Vongsamphanh, R., Yang, X., Yang, S., Ramotar, D., 2008. Human glyceraldehyde-3-phosphate dehydrogenase plays a direct role in reactivating oxidized forms of the DNA repair enzyme APE1. J. Biol. Chem. 283, 30632-30641.

Brinkkötter, A., Shakeri-Garakani, A., Lengeler. J.W., 2002. Two class II D-tagatosebisphosphate aldolases from enteric bacteria. Arch. Microbiol. 177, 410-419.

Butland, G., Peregrin-Alvarez, J.M., Li, J., Yang, W., Yang, X., Canadien, V., Starostine, 
A., Richards, D., Beattie, B., Krogan, N., Davey, M., Parkinson, J., Greenblatt, J., Emili, A., 2005. Interaction network containing conserved essential protein complexes in Escherichia coli. Nature, 433(7025), 531-537.

Cabiscol, E., Ros, J., 2006. From protein modifications to cellular dysfunction and diseases, in: Redox proteomics. Jonh Wiley \& Sons, Inc., New Jersey, USA.

Casadaban, M.J., 1976. Transposition and fusion of the lac genes to selected promoters in Escherichia coli using bacteriophage lambda and Mu. J. Mol. Biol. 104, 541-555.

Colussi. C., Albertini, M.C., Coppola, S., Rovidati, S., Galli, F., Ghibelli, L., 2000. $\mathrm{H}_{2} \mathrm{O}_{2}$ Induced block of glycolisis as an active ADP-ribosylation reaction protecting cells from apoptosis. FASEB J. 14, 2266-2276.

Deuerling E., Schulze-Specking, A., Tomoyasu, T., Mogk, A., Bukau, B., 1999. Trigger factor and DnaK cooperate in folding of newly synthesized proteins. Nature 400, 693-696.

Deuerling E., Patzelt, H., Vorderwülbecke, S., Rauch, T., Kramer, G., Schaffitzel, E., Mogk, A., Schulze-Specking, A., Langen, H., Bukau, B., 2003. Trigger factor and DnaK possess overlapping substrate pools and binding specificities. Mol. Microbiol. 47, 1317-28.

Eaton, P., Wright, N., Hearse, D.J., Shattock, M.J., 2002. Glyceraldehyde-3-phosphate dehydrogenase oxidation during cardiac ischemia and reperfusion. J. Mol. Cell. Cardiol. 34, 1549-1560.

Egea, L., Aguilera, L., Gimenez, R. , Sorolla, M.A., Aguilar, J., Badía, J., Baldomà, L., 2007. Role of secreted glyceraldehyde-3-phosphate dehydrogenase in the infection mechanism of enterohemorrhagic and enteropathogenic Escherichia coli: interaction 
of the extracellular enzyme with human plasminogen and fibrinogen. Int. J. Biochem. Cell Biol. 39, 1190-1203.

Herberg, C., Flórez-Weideinger, L. A., Dörrbecker, B., Hübner, S., Stülke, J., Commichau, F.M., 2007. SPINE: A method for the rapid detection and analysis of proteinprotein interactions in vivo. Proteomics 7, 4032-4035.

Huang, G-C., Li, Z-Y. Zou, Y-M., Fisher, G., 2000. Assisted folding of D-glyceraldehyde3-phosphate dehydrogenase by trigger factor. Protein Sci. 9, 1254-1261.

Jeffery, C.J., 2004. Molecular mechanisms for multitasking: recent crystal structures of moonlighting proteins. Curr. Opin. Struc. Biol. 14, 663-668.

Jeffery, C.J., 2009. Moonlighting proteins - an update. Mol. Biosyst. 5, 345-350.

Jia, Y., Xue, L., Liu, H., Li, J., 2009. Characterization of the glyceraldehyde-3-phosphate dehydrogenase (GAPDH) gene from the halotolerant alga Dunaliella salina and inhibition of its expression by RNAi. Curr. Microbiol. 58, 426-431.

Jin, H., Song, Y. P., Boel, G., Kochar, J., Pancholi, V., 2005. Group A streptococcal surface GAPDH, SDH, recognizes uPAR/CD87 as its receptor on the human pharyngeal cell and mediates bacterial adherence to host cells. J. Mol. Biol. 350, 2741.

Jin, H., Agarwal, S., Agarwal S., Pancoli, V., 2011. Surface export of GAPDH/SDH, a glycolytic enzyme, is essential for Streptococcus pyogenes virulence. mBio 2(3)e00068-11.

Kitagawa, M., Ara, T., Arifuzzaman, M., Ioka-Nakamichi, T., Inamoto, E., Toyonaga, H., Mori, H., 2005. Complete set of ORF clones of Escherichia coli ASKA library (a complete set of E. coli K-12 ORF archive): unique resources for biological research. DNA Res. 12, 291-299. 
584 Laemmli, U.K., 1970. Cleavage of structural proteins during the assembly of the head of 585 bacteriphage T4. Nature 222, 680-685.

586 Lowry, O.H., Rosebrough, N.J., Farr, A.L., Randall, R.J., 1951. Protein measurement 587 with the Folin Phenol reagent. J. Biol. Chem. 193, 265-273.

588 Pancholi, V., Chhatwal, G.S., 2003. Housekeeping enzymes as virulence factors for 589 pathogens. Int. J. Med. Microbiol. 293, 391-401.

590 Pellicer, M.T., Nuñez, M.F., Aguilar, J., Badia, J., Baldomà, L., 2003. Role of 2591 phosphoglycolate phosphatase of Escherichia coli in metabolism of the 2592 phosphoglycolate formed in DNA repair. J. Bacteriol. 185, 5815-5821.

593 Povirk, F.L., 1996. DNA damage and mutagenesis by radiomimetic DNA cleaving agents: 594 bleomycin, neocarzinostatin and others enediynes. Mutat. Res. 355, 71-89.

595 Sambrook, J., Rusell, D.W., 2001. Molecular cloning: A laboratory manual. Cold Spring $596 \quad$ Harbor Laboratory. Cold Spring Harbor, New York.

597 Sánchez, B., Schmitter, J.M., Urdaci, M.C., 2009a. Identification of novel proteins 598 secreted by lactobacillus plantarum that bind to mucin and fibronectin. J. Mol. $599 \quad$ Microbiol. Biotechnol. 17, 158-162.

600 Sánchez, B., Schmitter, J.M., Urdaci, M.C., 2009b. Identification of novel proteins 601 secreted by Lactobacillus rhamnosus GG grown in the Mann-Rogosa-Sharpe broth.

603 Schaumburg, J., Diekmann, O., Hagendorf, P., Bergmann, S., Rodhe, M., Hammerschmidt, 604 S., Jänsch, L., Wehland, J., Kärst, U., 2004. The cell wall proteome of Listeria 605 monocytogenes. Proteomics 4, 2991-3006.

606 Seifert, K.N., McArthur, W. P., Bleiweis, A. S., Brady, J., 2003. Characterization of 607 group B streptococcal glyceraldehyde-3-phosphate dehydrogenase: surface 
608

609

610

611

612

613

614

615

616

617

618

619

620

621

622

623

624

625

626 localization, enzymatic activity, and protein-protein interactions. Can. J. Microbiol. 49, 350-356.

Sinz, A., 2010. Investigation of ptotein-protein interactions in living cells by chemical crosslinking and mass spectrometry. Anal. Bioanal. Chem. 397, 3433-3440.

Sirover, M.A., 1999. New insights into an old protein: the functional diversity of mammalian glyceraldehyde-3-phosphate dehydrogenase. Biochim. Biophys. Acta 1432, 159-184.

Sirover, M.A., 2005. New nuclear functions of the glycolytic protein, glyceraldehyde-3phosphate dehydrogenase, in mammalian cells. J. Cell. Biochem. 95, 45-52.

Sirover, M.A., 2011. On the functional diversity of glyceraldehyde-3-phosphate dehydrogenase: biochemical mechanisms and regulatory control. Biochim. Biophys. Acta 1810, 741-751.

Taga, M.E., 2007. Bacterial signal destruction. ACS Chem. Biol. 2, 89-92.

Vasilescu, J., Guo, X., Kast, J., 2004. Identification of protein-protein interactions using in vivo cross-linking and mass spectrometry. Proteomics 4, 3845-3854.

Xavier, K.B., Miller, S.T., Lu, W., Jeong, K., Rabonowitz, J., Pelczer, I., Semmel-Hack, M.F.,Bassler, B.L., 2007. Phosphorylation and processing of the quorum-sensing molecule AI-2 in enteric bacteria. ACS Chem. Biol. 2, 128-136. 
627

628

629

630

631

632

633

634

635

636

637

638

639

640

641

642

643

644

645

646

647

648

649

\section{Legends to figures}

Fig. 1. In vivo crosslinking of GAPDH-V5. TOP10 cells expressing GAPDH-V5 were incubated with $1 \%$ formaldehyde for $20 \mathrm{~min}(\mathrm{FA}+)$ or left untreated (FA -). TOP10 cells without the recombinant plasmid pBAD-GapA were processed in parallel as control. (A) Cell extracts were analyzed by Western blotting with anti-V5 or anti-GAPDH specific antibodies. (B) Cell extracts obtained from TOP10 cells harbouring plasmid pBAD-GapA after formaldehyde cross-linking were incubated at the indicated conditions to reverse cross-links. Reversion was analyzed by Western blotting with anti-V5 antibodies.

Fig. 2. Immunoaffinity purification of GAPDH-V5 interacting proteins. (A) Purification of GAPDH-V5 interacting proteins after formaldehyde cross-linking. TOP10 cells expressing GAPDH-V5 were incubated with 1\% formaldehyde for 20 min (+ FA) or left untreated (FA). GAPDH-V5 complexes were purified from the corresponding cell extracts under astringent conditions using anti-V5 agarose beads. The purified complexes were incubated at $95^{\circ} \mathrm{C}$ for 30 min to reverse cross-links and then separated by SDS-PAGE. Proteins were visualized by Sypro ${ }^{\circledR}$ Ruby staining. Twelve protein bands visible in the + FA lane (labelled by white numbers) were excised from the gel and analyzed by MS. M, molecular mass marker. (B) Two-dimensional analysis of proteins immunopreciptated with GAPDHV5 in the absence of formaldehyde cross-linking. Proteins were visualized by Sypro® Ruby staining. Ten spots (labelled by white numbers) were excised from the gel and analyzed by MS. 
651 Fig. 3. Pull-down experiments showing binding of GAPDH to the ATP synthase $\alpha$ (AtpA)

652 and $\beta$ (AtpD) subunits. Cell extracts obtained from induced cells of strain AG1 bearing 653 ASKA clone JW3710 (expressing His $_{6}$-AtpD) or ASKA clone JW3712 (expressing His $6^{-}$

654 AtpA) were applied to a $\mathrm{Ni}^{2+}$-NTA column. After extensive washing, elution of

655 recombinant proteins was achieved with $300 \mathrm{mM}$ imidazole (elution buffer). As a control,

656 AG1 cell extract was applied to the $\mathrm{Ni}^{2+}$-NTA column and processed in parallel (right

657 panel). Column fractions (FT: flow-through; W: wash; E: elution) were analyzed either by

658 Coomassie blue staining (upper panel) or by Western blot with anti-GAPDH antibodies.

659

660 Fig. 4. Binding of GAPDH to Gph. (A) Pull-down experiments showing binding of

661 GAPDH to Gph. Cell extracts $(0.5 \mathrm{ml} ; 10 \mathrm{mg} / \mathrm{ml})$ obtained from induced cells of strain

662 BLB21(DE3) bearing plasmid pGEX (expressing GST) or plasmid pGEX-Gph (expressing

663 GST-Gph) were passed over a glutathione-sepharose 4B column. After extensive washing,

664 elution of GST or GST-Gph was achieved with glutathione elution buffer. Column

665 fractions (FT: flow-through; W: wash; E: elution) were analyzed either by Coomassie blue

666 staining (upper panel) or by Western blot with anti-GAPDH antibodies. (B) Co-purification

667 of GAPDH with Gph. Gph was purified by affinity chromatography from induced cell

668 extracts of strain BLB21(DE3) bearing plasmid pGEX-Gph (expressing GST-Gph). In this

669 case, elution of Gph was achieved by factor Xa digestion. The eluted fractions were

670 analyzed either by Coomassie blue staining (upper panel) or by Western blot with anti-

671 GAPDH antibodies. The $35 \mathrm{kDa}$ protein band was excised from the stained gel and

672 identified as GAPDH by MS. (C) Far-Western analysis of binding of soluble Gph to

673 PVDF-immobilized GAPDH. Samples containing $5.0 \mu \mathrm{g}$ of purified GAPDH were 
674 subjected to 2D gel electrophoresis, and the gel was either silver-stained (first panel) or

675 electroblotted. The PVDF membrane was reacted with purified Gph (5 $\mu \mathrm{g} / \mathrm{ml})$,

676 subsequently incubated with antibodies against anti-Gph and processed to visualize the

677 reactive bands (second panel). The third panel shows the result of this analysis when the

678 incubation step with Gph was omitted.

679

680 Fig. 5. Pull-down experiments to analyze interaction of GAPDH with Gph in cells

681 challenged with bleomycin. Induced cells of strain JA210 bearing plasmid pGEX-Gph

682 (expressing GST-Gph) were challenged with $8 \mu \mathrm{g} / \mathrm{ml}$ bleomycin-50 $\mu \mathrm{M}$ de $\mathrm{FeSO}_{4}$. At the

683 indicated times, cell extracts were obtained. Samples were incubated with glutathione-

684 sepharose 4B resin. After extensive washing, elution of GST-Gph was achieved with

685 loading buffer $1 x$. Fractions were analyzed by Coomassie blue staining and by Western blot

686 with anti-GAPDH or anti-GST antibodies. 
687 Table 1.

688 Proteins that copurified with GAPDH-V5 after in vivo formaldehyde cross-linking

\begin{tabular}{cllccccc}
\hline Band & \multicolumn{1}{c}{ Protein } & Accession & kDa & $\begin{array}{c}\text { MASCOT } \\
\text { score }\end{array}$ & $\begin{array}{c}\text { Peptides } \\
\text { sequenced }\end{array}$ & Function & $\begin{array}{c}\text { Subcellular } \\
\text { localization }\end{array}$ \\
\hline 1 & D-ribose binding protein (RbsB) & P02925 & 28.5 & 236 & 5 & D-ribose transport & Periplasm \\
1 & Phosphoglycolate phosphatase & P32662 & 27.4 & 99 & 3 & Metabolic enzyme & Cytoplasm \\
2 & GAPDH & P0A9B2 & 36.1 & 447 & 11 & Metabolic enzyme & Cytoplasm \\
2 & OmpC & P06996 & 40.4 & 91 & 3 & Porin & Outer membrane \\
3 & GAPDH-V5 & -- & -- & 347 & 12 & Recombinant protein & -- \\
4 & EF-Tu & P0CE47 & 43.3 & 503 & 14 & Protein synthesis & Cytoplasm \\
5 & Enolase & P0A6P9 & 45.6 & 238 & 5 & Metabolic enzyme & Cytoplasm \\
6 & Tryptophanase & P0A853 & 53.1 & 183 & 5 & Metabolic enzyme & Cytoplasm \\
6 & ATP synthase $\beta$ subunit & P0ABB4 & 50.3 & 98 & 3 & ATP synthesis & Inner membrane \\
7 & Aldehyde dehydrogrenase (AldA) & P25553 & 52.4 & 284 & 6 & Metabolic enzyme & Cytoplasm \\
7 & Trigger factor & P0A850 & 49.0 & 115 & 4 & Chaperone & Cytoplasm \\
7 & Pyruvate kinase & P0AD61 & 51.6 & 97 & 3 & Metabolic enzyme & Cytoplasm \\
8 & Non identified & -- & -- & -- & -- & -- & - \\
9 & DnaK & P0A6Y8 & 69.1 & 510 & 10 & Chaperone & Cytoplasm \\
10 & Transketolase & P27302 & 72.4 & 105 & 5 & Metabolic enzyme & Cytoplasm \\
11 & Aconitase & P25516 & 94.0 & 135 & 3 & Metabolic enzyme & Cytoplasm \\
12 & Non identified & -- & -- & -- & -- & & - \\
& & & & & & -
\end{tabular}




\begin{tabular}{|c|c|c|c|c|c|c|c|c|}
\hline Spot & Protein & Accesion & $\mathrm{kDa}$ & $\mathrm{pI}$ & Score & $\begin{array}{l}\text { Peptides } \\
\text { sequenced }\end{array}$ & Function & $\begin{array}{l}\text { Subcellular } \\
\text { localization }\end{array}$ \\
\hline 1 & ATP synthase $\alpha$ subunit & P0ABB0 & 55.2 & 6.13 & 608.59 & 29 & ATP synthesis & $\begin{array}{l}\text { Membrane } \\
\text { bound }\end{array}$ \\
\hline 1 & Anthranilate synthase, component II & P00904 & 56.8 & 6.52 & 74.04 & 10 & Trp biosynthesis & Cytoplasm \\
\hline 1 & ATP-binding protein AraG & P0AAF3 & 55.0 & 6.38 & 59.09 & 10 & L-Arabinose transport & Cytoplasm \\
\hline 1 & ATP-binding protein RbsA & P04983 & 55.0 & 6.14 & 50.91 & 11 & Function & Cytoplasm \\
\hline 1 & Dihydrolipoyl dehydrogenase (lpdA) & P0A9P0 & 50.7 & 6.15 & 49.10 & 11 & Pyruvate DH component & Cytoplasm \\
\hline 2 & Non-identified & -- & -- & -- & -- & -- & -- & -- \\
\hline 3 & ATP synthase $\beta$ subunit & P0ABB4 & 50.3 & 5.01 & 1150 & 40 & ATP synthesis & $\begin{array}{l}\text { Membrane } \\
\text { bound }\end{array}$ \\
\hline 4 & OmpF & Р02931 & 39.3 & 4.96 & 156.9 & 19 & Porin & Outer membrane \\
\hline 5 & OmpC & P06996 & 40.3 & 4.82 & 445.05 & 31 & Porin & Outer membrane \\
\hline 5 & GAPDH & P0A9B2 & 35.5 & 7.11 & 153.96 & 19 & Metabolic enzyme & Cytoplasm \\
\hline 6 & GAPDH & P0A9B2 & 35.5 & 7.11 & 629.11 & 29 & Metabolic enzyme & Cytoplasm \\
\hline $7 / 9 / 10$ & GAPDH proteolysis fragments & P0A9B2 & -- & -- & -- & -- & -- & -- \\
\hline 8 & $\begin{array}{l}\text { D-tagatose-1,6-bisphosphoaldolase, } \\
\text { GatY }\end{array}$ & P0C8J6 & 30.8 & 6.34 & 259.55 & 21 & Metabolic enzyme & Cytoplasm \\
\hline
\end{tabular}




\section{FIGURE 1}

(A)

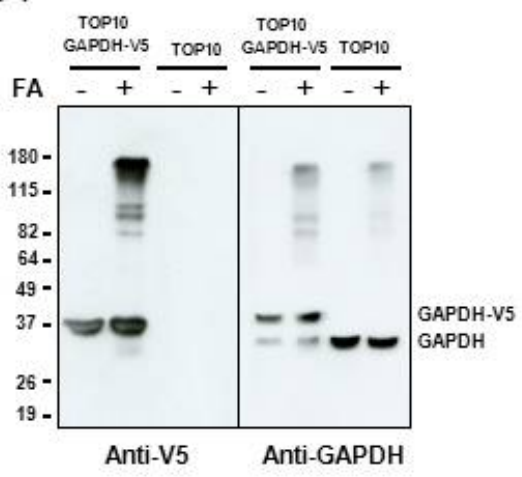

(B)

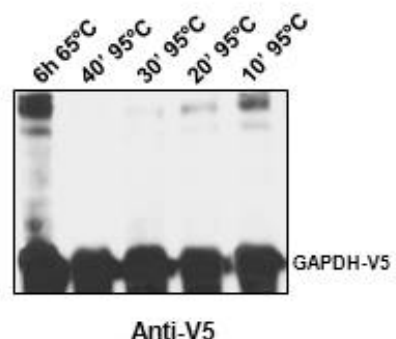

Anti-V5 
FIGURE 2

(A)

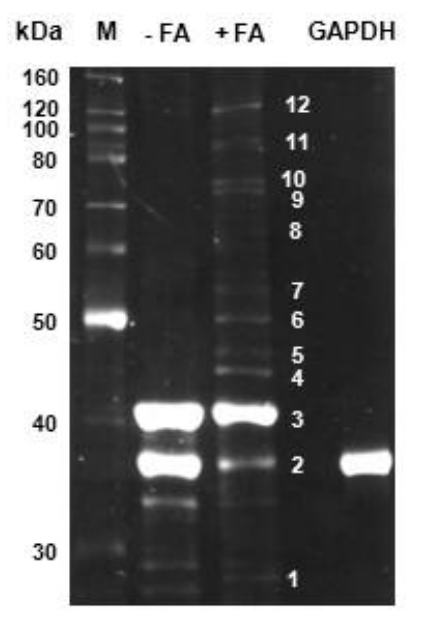

(B)

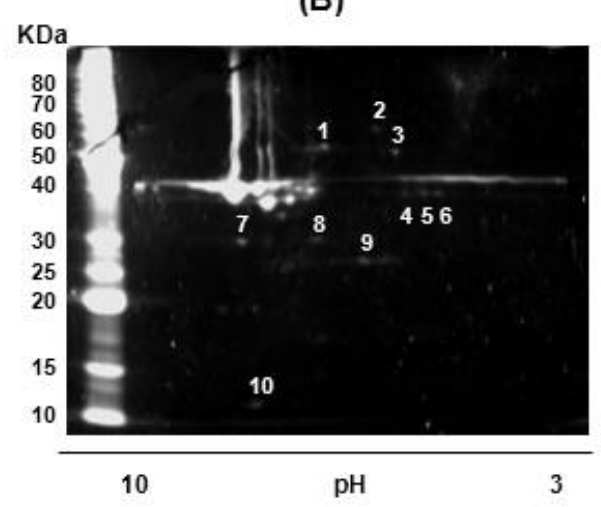


FIGURE 3

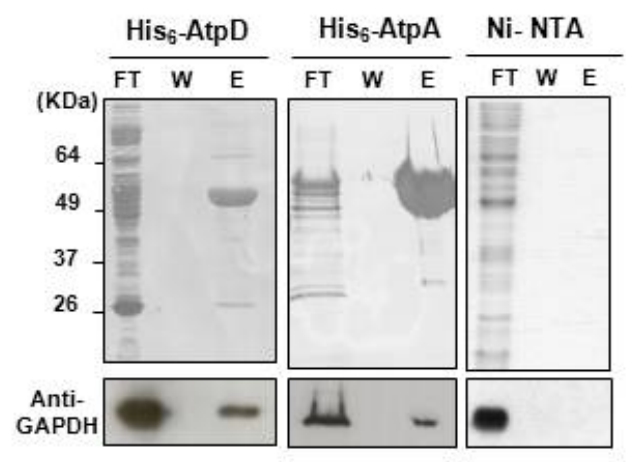


FIGURE 4

(A)

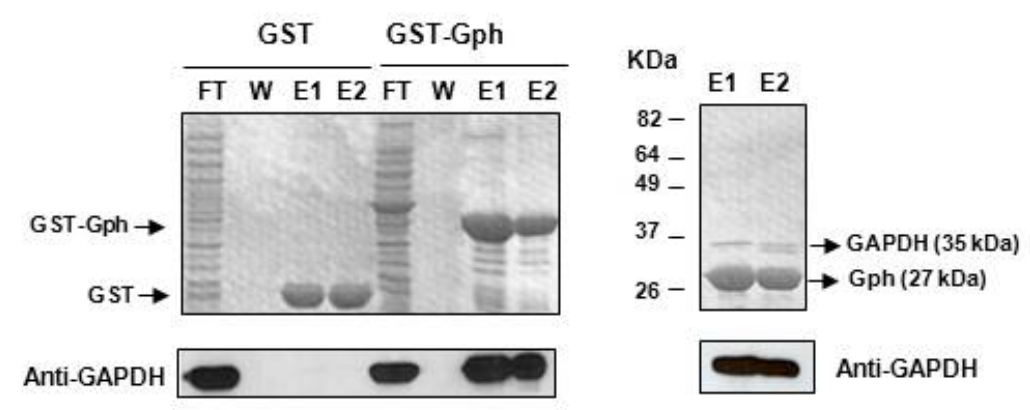

(C)

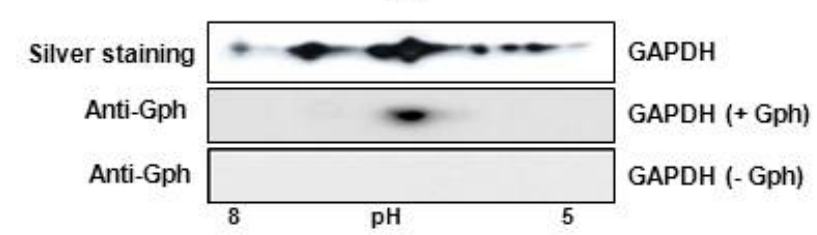


FIGURE 5

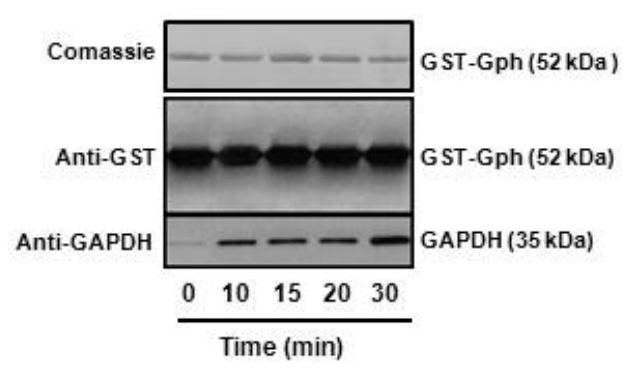

